\title{
Telecom-Band Quantum Optics with Ytterbium Atoms and Silicon Nanophotonics
}

\author{
Jacob P. Covey, ${ }^{1, *}$ Alp Sipahigil, ${ }^{2}$ Szilard Szoke, ${ }^{2}$ Neil Sinclair, ${ }^{1,3}$ Manuel Endres, ${ }^{1}$ and Oskar Painter ${ }^{2}$ \\ ${ }^{1}$ Division of Physics, Mathematics and Astronomy, California Institute of Technology, Pasadena, \\ California 91125, USA \\ ${ }^{2}$ Thomas J. Watson, Sr., Laboratory of Applied Physics, California Institute of Technology, Pasadena, \\ California 91125, USA \\ ${ }^{3}$ Alliance for Quantum Technologies, California Institute of Technology, Pasadena, \\ California 91125, USA
}

(Received 30 October 2018; revised manuscript received 16 January 2019; published 19 March 2019)

\begin{abstract}
Wavelengths in the telecommunication window (approximately 1.25-1.65 $\mu \mathrm{m}$ ) are ideal for quantum communication due to low transmission loss in fiber networks. To realize quantum networks operating at these wavelengths, long-lived quantum memories that couple to telecom-band photons with high efficiency need to be developed. We propose coupling neutral ytterbium atoms, which have a strong telecomwavelength transition, to a silicon photonic crystal cavity. Specifically, we consider the ${ }^{3} P_{0} \leftrightarrow{ }^{3} D_{1}$ transition in neutral ${ }^{171} \mathrm{Yb}$ to interface its long-lived nuclear spin in the metastable ${ }^{3} P_{0}$ "clock" state with a telecom-band photon at $1.4 \mu \mathrm{m}$. We show that $\mathrm{Yb}$ atoms can be trapped using a short-wavelength (approximately $470 \mathrm{~nm}$ ) tweezer at a distance of $350 \mathrm{~nm}$ from the silicon photonic crystal cavity. At this distance, due to the slowly decaying evanescent cavity field at a longer wavelength, we obtain a singlephoton Rabi frequency of $g / 2 \pi \approx 100 \mathrm{MHz}$ and a cooperativity of $C \approx 47$ while maintaining a high photon collection efficiency into a single mode fiber. The combination of high system efficiency, telecomband operation, and long coherence times makes this platform well suited for quantum optics on a silicon chip and long-distance quantum communication.
\end{abstract}

DOI: 10.1103/PhysRevApplied.11.034044

\section{INTRODUCTION}

Efficient interfaces between single atoms and single photons could enable long-distance quantum communication based on quantum repeaters [1-7] and constitute a novel platform for many-body physics with long-range interactions [8,9]. While most atom-photon interfaces to date operate at visible or near-infrared wavelengths (approximately 700-1000 nm), compatibility with telecom wavelengths (approximately 1.25-1.65 $\mu \mathrm{m}$ ) is highly desired, both for quantum communication due to low propagation loss in fiberoptic cables and for compatibility with silicon-based photonics. Accordingly, most approaches to quantum communication require frequency conversion of single photons into the telecom window, which often results in additional noise photons and reduced efficiencies [10-12]. A platform combining both long atomic coherence times and high emission bandwidth at telecom wavelengths has yet to be developed.

Atomlike defects in solids [13-16] and trapped neutral atoms [17-21] coupled to photonic crystal cavities

\footnotetext{
*covey@caltech.edu
}

hold a promise to achieve such light-matter interactions. Atomlike defects in solids require no external trapping potential since they are held in the crystal field of the host solid-state environment. However, this environment has drawbacks, such as inhomogeneous broadening, phonon broadening, and spectral diffusion [22-24]. Hence, these systems require cooling to cryogenic temperatures to reduce phonon broadening and spectral tuning to achieve indistinguishability [13]. Moreover, the atomlike defects investigated to date are outside of the telecom window, have short coherence times, or have low emission bandwidths $[22,24]$.

Optically trapped atoms in free space offer the prospect of significantly improved coherence properties since inhomogeneous broadening and spectral diffusion are negligible. However, an outstanding challenge is to reliably trap an atom sufficiently close to a photonic device. Previous trapping efforts were based on evanescent fields that confine the atom near the device $[17,25,26]$ or were carried out by forming a standing wave trap via reflection from the device $[18,27]$. Surface effects such as van der Waals forces [28,29], surface patch charges [30], and Casimir-Polder forces [31-33] complicate these 
approaches. Moreover, the emission wavelengths of the atomic species used to date - rubidium and cesium - are approximately $800 \mathrm{~nm}$, well outside the telecom window.

\section{OVERVIEW OF THE SYSTEM}

We propose a platform based on short-wavelength optical tweezer trapping [34-36] to hold an $\mathrm{Yb}$ atom near a silicon photonic crystal in order to obtain strong atom-cavity interactions. The atomic transition is from the metastable "clock" state and has a wavelength of $\lambda=1.4 \mu \mathrm{m}$. Compared to previous work with tweezers operating at $\lambda_{\text {twzr }} \approx$ $800 \mathrm{~nm}$ [19], we use $\lambda_{\text {twzr }} \approx 470 \mathrm{~nm}$ to obtain tighter focusing. Further, the use of an approximately $2 \times$ longer wavelength transition results in a larger spatial extent of the evanescent cavity field. Accordingly, we propose to trap the atom without the use of reflection from the device. We use a larger distance from the device compared to previous work [36] $\left(d_{\mathrm{twzr}}=350 \mathrm{~nm}\right)$, at which surface forces are reduced by a factor of $>10$. The larger disparity between the trapping and the telecom-transition wavelengths in $\mathrm{Yb}$ enables both a fivefold increase in cooperativity and more robust atom trapping.

We focus on quantum communication as a specific application of this platform and we envision an $\mathrm{Yb}$ atom coupled to a silicon nanophotonic cavity as a node in a quantum repeater network (Fig. 1). To this end, we propose a partially open cavity design which enables the emission of approximately 15-MHz-bandwidth photons entangled with the nuclear spin of ${ }^{171} \mathrm{Yb}$ that serves as a long-lived quantum memory. Further, we consider a fiber gap FabryPerot cavity in Appendix C rather than a photonic crystal, which may offer a simpler alternative, but is not compatible with on-chip silicon photonics.

We highlight the use of silicon for the photonic crystal cavity not only due to low losses but also its maturity as a fabrication technology [37]. Robust and high-yield electronic, mechanical, and optical devices have been realized in silicon-based systems utilizing a wide array of highly developed micro- and nanofabrication techniques. Indeed, custom silicon devices are increasingly commercially available from fabrication foundries (see, e.g., Ref. [38]). Moreover, silicon is compatible with other photonic technologies [39] such as electro-optomechanical [40,41] and optomechanical $[42,43]$ systems.

The precise control of single atoms in our approach enables scalable extension to multiple atoms by employing recently demonstrated techniques with tweezer arrays [27, $44,45]$. This scalability would enable photonic coupling within an array of atoms, which could lead to a novel platform for many-body physics [46,47], quantum nonlinear optics [20,21], and photon-mediated quantum gates [48]. The latter application is relevant to quantum repeaters, where deterministic two-qubit gates in each node could enhance entanglement distribution rates by realizing

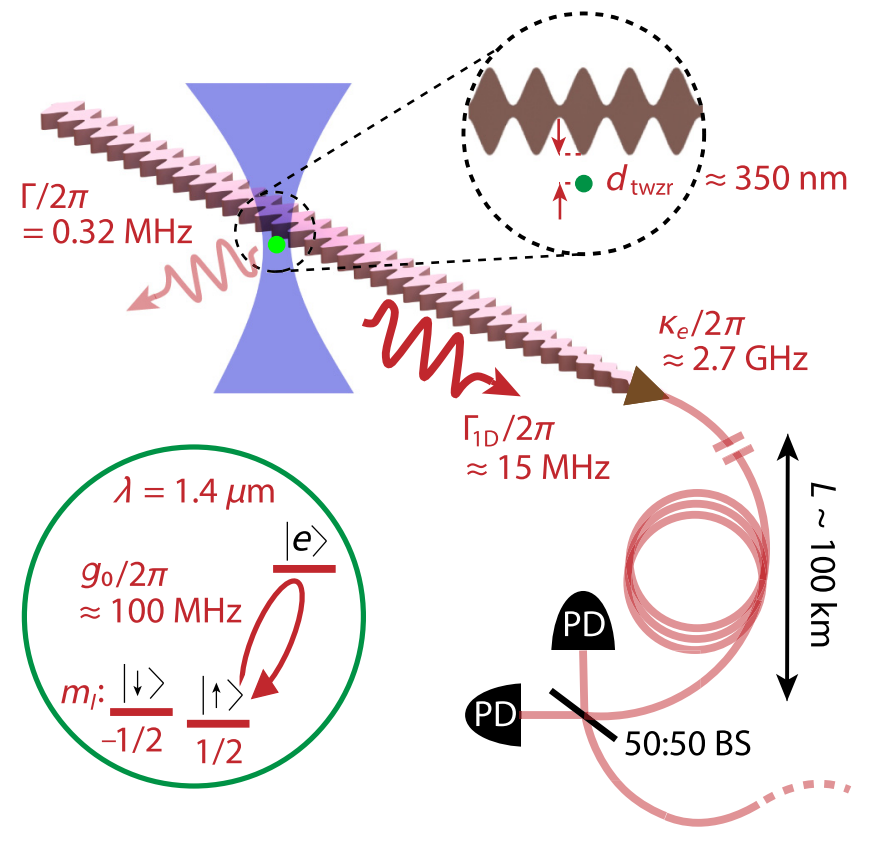

FIG. 1. Schematic overview. Silicon photonic crystal cavity with an ${ }^{171} \mathrm{Yb}$ atom trapped nearby in an optical tweezer. The minimum atom-device separation $d_{\mathrm{twzr}} \approx 350 \mathrm{~nm}$ allowed by our approach corresponds to an atom-cavity system on a strong telecom-band transition with vacuum Rabi frequency $g_{0} / 2 \pi \approx$ $100 \mathrm{MHz}$ and emission bandwidth of $\Gamma_{1 \mathrm{D}} / 2 \pi \approx 15 \mathrm{MHz}$, for a partially open cavity with external coupling $\kappa_{e} / 2 \pi \approx 2.7 \mathrm{GHz}$ and atomic free-space linewidth of $\Gamma / 2 \pi=0.32 \mathrm{MHz}$. The nuclear spin projections $m_{I}$ are of the $I=1 / 2$ nuclear spin of ${ }^{171} \mathrm{Yb}$. The photon in the cavity is coupled to an optical fiber with length $L \sim 100 \mathrm{~km}$. This system constitutes a node in a telecom quantum repeater in which entanglement between nodes is established by a Bell state measurement using a 50:50 beam splitter (BS) and single-photon detectors (PD).

efficient Bell state measurements for entanglement swapping operations. Note that two-qubit gates could also be accomplished using local exchange [49-51] or Rydberg [52] interactions.

The strong telecom-wavelength transition of $\mathrm{Yb}$ is from a metastable state with lifetime $\tau \approx 26 \mathrm{~s}$ (Fig. 2), which is the crutial state in the optical clock transition [53]. We focus on the 1.4- $\mu \mathrm{m}\left({ }^{3} P_{0} \rightarrow{ }^{3} D_{1}\right)$ transition, which is shown with the orange double arrow (see Appendix A). Concerning the other transitions available, the one at 1.5 $\mu \mathrm{m}\left({ }^{3} P_{1} \rightarrow{ }^{3} D_{1}\right)$ is hampered by the short lifetime of ${ }^{3} P_{1}$, which restricts its use to more complex protocols. Finally, the $2.1-\mu \mathrm{m}$ transition $\left({ }^{3} P_{2} \rightarrow{ }^{3} D_{1}\right)$ is not suitable for fiberoptic communication. However, it is an interesting candidate for free-space communication given the relatively high atmospheric transmission at this wavelength. We define the states of interest as $|\downarrow\rangle \equiv{ }^{3} P_{0} \mid F=$ $\left.1 / 2, m_{F}=-1 / 2\right\rangle,|\uparrow\rangle \equiv{ }^{3} P_{0}\left|F=1 / 2, m_{F}=1 / 2\right\rangle$, and $|e\rangle \equiv{ }^{3} D_{1}\left|F=3 / 2, m_{F}=3 / 2\right\rangle$ (see Figs. 1 and 3 ). Note that care must be taken to correctly account for mode 


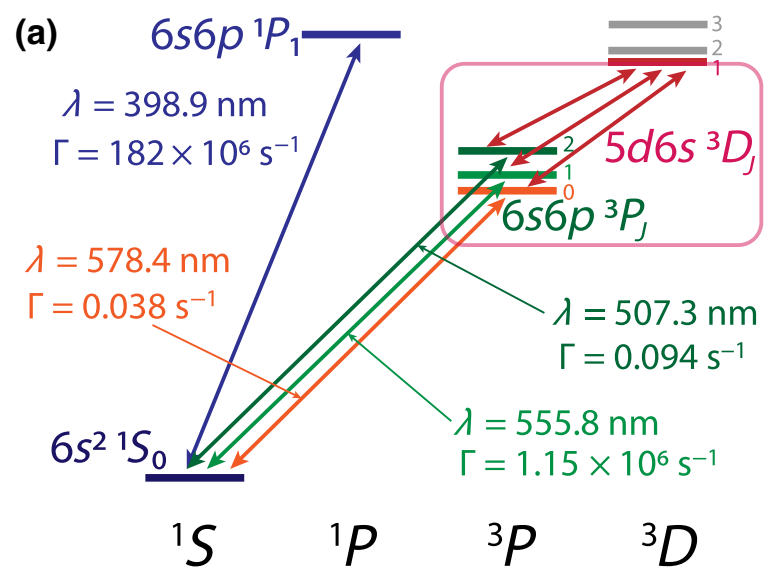

(b)

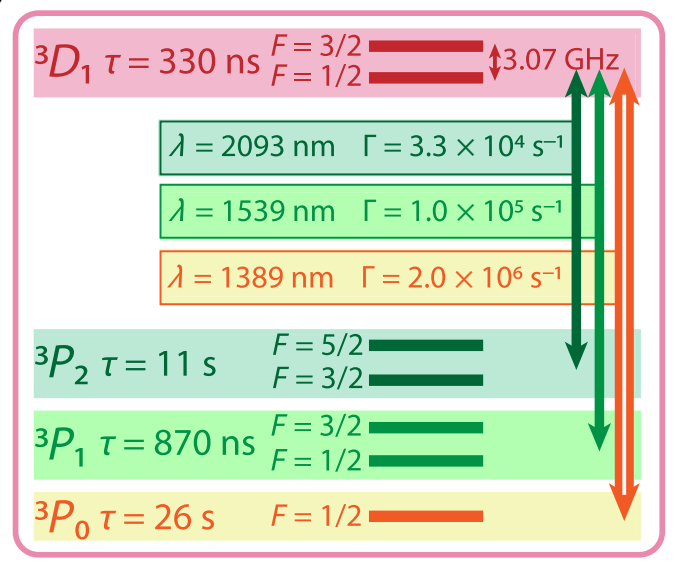

FIG. 2. Level diagram of the relevant states of ${ }^{171} \mathrm{Yb}$. (a) Low-lying states of $\mathrm{Yb}$ in the singlet and triplet manifolds. The telecom transitions from the metastable $6 s 6 p{ }^{3} P_{J}$ states to the $5 d 6 s^{3} D_{1}$ state are highlighted in the red box. (b) Enlargement of the highlighted transitions. The nuclear spin in ${ }^{171} \mathrm{Yb}$ is $I=1 / 2$, so the hyperfine states are given by $F=1 / 2$ when $J=0$ and $F=\{J+1 / 2, J-1 / 2\}$ when $J \geq 1$. The lifetimes of the ${ }^{3} P_{J}$ states, transition wavelengths, and transition linewidths, as well as the lifetime and hyperfine splitting of the ${ }^{3} D_{1}$ state, are given. We employ the transition shown with the orange double arrow.

overlap with the cavity field, particularly in the case of circularly polarized emission (see Appendix A).

\section{APPLICATION IN A QUANTUM REPEATER}

Before providing a detailed description of the photonic cavity and the coupling of the atom, we briefly highlight the potential of this platform for quantum communication (see Appendix B for more details). As an example, we consider utilizing our system in a quantum repeater architecture by entangling an ${ }^{171} \mathrm{Yb}$ nuclear spin and a telecom photon using the Barret-Kok scheme with time-bin photonic qubits $[54,55]$. The key parameters of our system that impact the entanglement generation rate and fidelity are summarized in Table I. We describe in detail in the

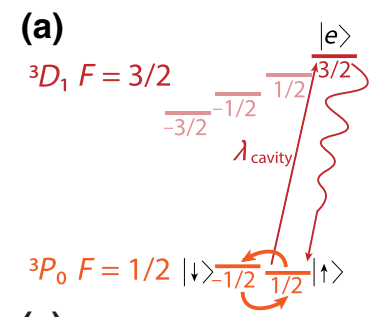

(c)

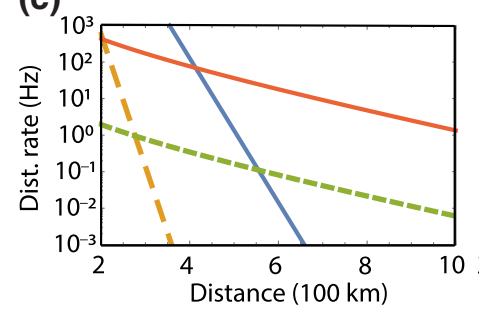

(d)

(b)
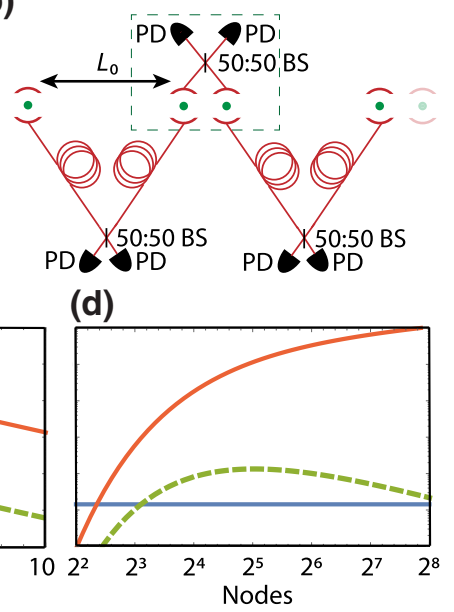

FIG. 3. Spin-photon entanglement scheme and quantum repeater operation. (a) The relevant (irrelevant) hyperfine states are shown in solid (semitransparent) colors. The cavity-enhanced transition wavelength is $\lambda_{\text {cavity. }}$ (b) A single trapped ${ }^{171} \mathrm{Yb}$ atom in a cavity is represented by a green dot inside two curved semicircles. Local node pairs are shown in the dashed box. Node pairs are separated by $L_{0}$. BS denotes the beam splitter and PD the single-photon detector. (c) The entanglement distribution rate versus total distance via direct communication at $10 \mathrm{GHz}$ for $1550 \mathrm{~nm}$ (solid blue curve) and $1390 \mathrm{~nm}$ (large-dashed orange) and via a quantum repeater with $2^{4}$ nodes with (without) local deterministic entanglement, shown as a solid red (short-dashed green) curve. Note that a $10-\mathrm{GHz}$ rate for the direct transmission scheme [62] can be interpreted as an information-theoretic bound for information distribution without quantum repeaters if an ideal single-photon source with a $10 / 1.44=6.9-\mathrm{GHz}$ repetition rate is employed [63]. (d) The entanglement distribution rate over $600 \mathrm{~km}$ versus the number of nodes via direct communication at $10 \mathrm{GHz}$ for $1550 \mathrm{~nm}$ (solid blue) and $1390 \mathrm{~nm}$ (large-dashed orange, not visible) and via a quantum repeater with (without) local deterministic entanglement, shown as a solid red (short-dashed green) curve.

following sections how these values are achieved in our platform.

Our system allows a bandwidth of $\Gamma_{1 \mathrm{D}}=2 \pi \times 15$ $\mathrm{MHz}$, which is sufficiently high to not limit the performance of the repeater. High bandwidth emission increases the detection fidelity since the acquisition time is reduced and the detection of dark counts can be mitigated. While

TABLE I. The values relevant for a quantum repeater with a photonic crystal cavity and the sections of the text in which they are described.

\begin{tabular}{lcc}
\hline \hline Parameter & Section & Value \\
\hline Bandwidth & IV & $2 \pi \times 15 \mathrm{MHz}$ \\
Wavelength & II & $1.39 \mu \mathrm{m}(0.35 \mathrm{~dB} / \mathrm{km})$ \\
System efficiency & IV & 0.80 \\
Memory & II, Appendix B & $\leq 26 \mathrm{~s}$ \\
Readout fidelity & Appendix B & $>0.99$ \\
\hline \hline
\end{tabular}


most emitter platforms have sufficient bandwidth, many of the platforms operating in the telecom band, such as rare-earth ions in crystals, have slow emission rates. The bare linewidth of erbium (Er) ions, for instance, is $\Gamma / 2 \pi \approx$ $14 \mathrm{~Hz}$ [56] and, thus, large Purcell enhancement in high$Q$ nanophotonic cavities [57,58] is required to enhance the emission rate.

The long-lived memory of the nuclear spin qubit is one of the strengths of our platform. Some atomlike defects such as nitrogen-vacancy (NV) centers in diamond also have long memory [59,60], but their optical transitions are at visible wavelengths and are hampered by phonon broadening. Further, many of the solid-state systems whose optical transitions are in the telecom band have short memories [61]. In our system, the memory is assumed to be limited to the lifetime of the ${ }^{3} P_{0}$ state, though care must be taken to mitigate various heating mechanisms associated with tweezer trapping. A unique feature of alkaline-earth(-like) atoms is the possibility of cooling via electronic states while preserving coherence of the nuclear spin [50].

Another important characteristic for determining the quantum repeater performance is the system efficiency, which describes the probability that a photon emitted by the atom is acquired into the fiber network. This includes the coupling to the cavity, the extraction from the cavity into the waveguide, and the coupling to a fiber. All these values are described in Sec. IV and the total photon system efficiency is expected to be $\eta_{\text {tot }} \approx 0.80$.

As a concrete demonstration of the potential of this system, we calculate the entanglement distribution rate in a network as shown in Figs. 3(a) and 3(b), and we compare it to direct communication without repeaters (see Appendix B for analysis). For a repeater system of 16 nodes, we find that the distribution rate exceeds that of direct communication with a $10-\mathrm{GHz}$ single-photon source $[62,63]$ for a minimum total distance of $550 \mathrm{~km}$. The corresponding entanglement distribution rate is 0.1 $\mathrm{Hz}$. However, when local entanglement swapping at a node can be realized using two-qubit gates rather than probabilistic photon-detection-based schemes, the distribution rate could be enhanced to $25 \mathrm{~Hz}$. The distribution rate versus distance for 16 nodes is shown in Fig. 3(c) and the rate versus number of nodes for $600 \mathrm{~km}$ is shown in Fig. 3(d). These findings indicate that this platform is a competitive quantum repeater technology in the telecom band. Note that the fiber gap Fabry-Perot alternative also performs well (see Appendix C).

As a specific point of reference, we compare to a system with the same cavity QED parameters in Table I but operating at half the wavelength, in the visible band. Because of the large attenuation loss of approximately 3.5 $\mathrm{dB} / \mathrm{km}$, such a system would require frequency conversion into the telecom window. Typical conversion efficiencies realized in alkali-atom [64], trapped ion [11], and nitrogen-vacancy-center [12] systems are approximately $25 \%$. We account for this by including it in the overall system efficiency, which goes from 0.8 to 0.2 . Based on the equations in Appendix B, the corresponding entanglement distribution rate for 16 nodes becomes $110 \mathrm{nHz}(1.6 \mathrm{~Hz})$ in the case of probabilistic (deterministic) local entanglement swapping. This simple comparison shows the power of starting with a photon whose wavelength is in the telecom band, particularly when local entangling gates are not available. We also note that frequency conversion results in additional noise photons owing to the presence of a strong pump field, which can cause false photon coincidences and reduced entanglement fidelity.

\section{THE SILICON PHOTONIC CRYSTAL CAVITY}

We now describe the design of the partially open cavity and the resulting coupling strength to an $\mathrm{Yb}$ atom. We consider a photonic crystal geometry based on a nanobeam with an external corrugation [65]. The sinusoidal modulation along the outer edges induces a photonic band gap, which in turn enables the creation of a cavity via the introduction of a defect cell in the lattice to break the translational symmetry of the crystal. This enables the formation of modes localized in space around the defect region. For our chosen photonic crystal geometry, this is achieved by using a lattice constant $a_{\text {mirror }}=454 \mathrm{~nm}$. This is then subsequently tapered down to $a_{\text {cavity }}=433 \mathrm{~nm}$ such that the relevant band edge of the mirror region is tuned into the band gap and hence establishes a cavity region.

Details of the photonic crystal geometry are shown in Figs. 4(a) and 4(b). The different colors show the different sections of the cavity. From left to right, purple is the input section of the cavity that transitions from a single mode waveguide to the photonic crystal geometry, enabling the coupling of light in and out; the left blue section is the left cavity mirror with higher transmission; the left dark green section is the cavity taper region from the mirror cell lattice constant $a_{\text {mirror }}$ to the center cavity cell lattice constant $a_{\text {cavity }}$; the light green section is the central cavity unit cell; the right dark green section is the taper to the backside mirror; and right blue section is the backside mirror with very high reflectivity. The tapering is done in such a way as to produce an effective quadratic potential for localized cavity photons, providing the optimal balance between localization in the plane of the device and radiation out of plane [66-68]. The device thickness is chosen to be $100 \mathrm{~nm}$ to extend the evanescent field due to weaker confinement inside the dielectric, hence allowing for a greater distance between the photonic crystal and the atom.

Our design has a radiation limited quality factor of $3.5 \times 10^{6}$ in simulations. However, we anticipate that the quality factor will be limited by intrinsic fabrication imperfections to $Q_{i} \leq 7 \times 10^{5}$, whose corresponding intrinsic cavity linewidth is $\kappa_{i}=2 \pi \times 300 \mathrm{MHz}$ [69]. 
(a)
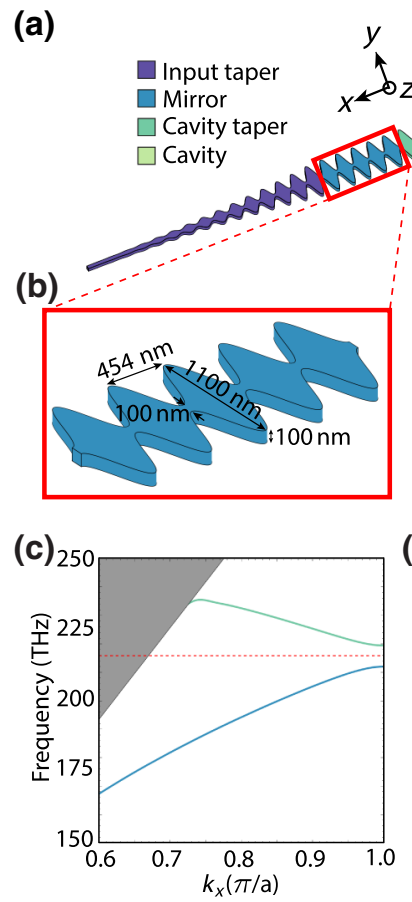

(d)

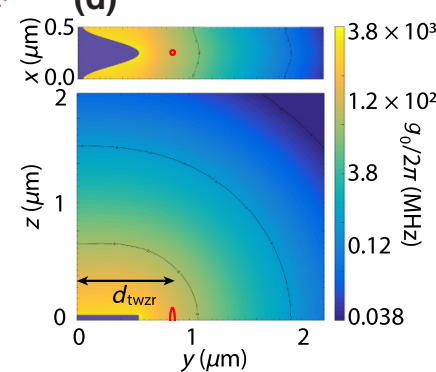

FIG. 4. Design and characterization of the Si cavity. (a) Schematic of the photonic crystal cavity. The different colors show the different sections of the cavity. (b) An enlargement of the first mirror section of the photonic cavity. (c) The TE mode band structure of the air (green) and dielectric (blue) mode. The red dashed line is the atomic resonance and the shaded gray region is outside the light cone. (d) The coherent coupling rate $g_{0}$ shown on a color map as a function of distance in the $y$ and $z$ directions from the center antinode of the photonic cavity $(x=0)$ in the bottom image, and the profile along the $x$ direction across the center tooth in the top image. The red ellipse and circle show the size of the atomic motional wave function (see Sec. V). (e) The blue left vertical scale shows the line cut of the coherent coupling rate for $x=z=0$ versus the distance from the surface. The dashed line represents the value of $g_{0}$ for which $C_{0}=1$. The green right vertical scale shows the surface force from the photonic crystal on the atom. The curve is meant to show the qualitative scaling only. The dashed line shows the maximum restoring force from an optical tweezer of depth $1 \mathrm{mK}$ and waist of approximately $330 \mathrm{~nm}$. The vertical red line shows the proposed position of the atom at $d_{\mathrm{twzr}}=350 \mathrm{~nm}$.

We design the cavity to be partially open on one side [as in Fig. 4(a)] to efficiently extract the cavity photons $[48,70,71]$. Specifically, we consider 5 mirror cells on the front mirror and 10 on the back mirror. The collection efficiency $\eta_{\text {coll }}$ of extracting the photon into the waveguide mode is given by $1-Q_{e} / Q_{i}$, where the subscript " $e$ " denotes external coupling. In our design, we choose a modest $Q_{e}=8 \times 10^{4}$, for which $\eta_{\text {coll }}=0.89$ and $\kappa_{e}=$ $2 \pi \times 2.7 \mathrm{GHz}$.

We now consider the photonic mode profile. The transverse electric (TE) photonic band structure containing a band gap centered on the atomic transition at $\lambda=1388.8$

$\mathrm{nm}(215.9 \mathrm{THz})$ is shown in Fig. 4(c). The evanescent field profile of the dielectric mode is shown in Fig. 4(d), where the simulated electric field per photon $E_{\text {cavity }}$ is converted to the vacuum Rabi frequency (i.e., coherent coupling rate) by $g_{0}=\mu E_{\text {cavity }} / \hbar$. $\mu$ is the dipole matrix element (DME) described in Appendix A. The red ellipse and circle show the $1 / e^{2}$ size of the atomic motional wave function, to be discussed in the next section. A line cut of the coherent coupling rate is shown in Fig. 4(e) for $x=z=0$ versus the distance from the surface, and we find an exponential length scale of $\lambda_{\mathrm{Si}} \approx 170 \mathrm{~nm}$, determined by fitting $g_{0}(d)=g_{0}(0) e^{-d / \lambda_{\mathrm{Si}}}$, where $d$ is the distance between the surface and the atom. The smoothness of the curve is limited by numerical resolution, but the data provide an accurate quantitative estimate over approximately 100-nm length scales. This is consistent with estimates for the mode dispersion based on the band structure [Fig. 4(c)]. The position of the atom is represented by the vertical red line.

The maximal evanescent coupling occurs at the surface of the photonic crystal, where the electric field per photon is $E_{\text {cavity }}=2.6 \times 10^{5} \mathrm{~V} / \mathrm{m}$. The coherent coupling rate can then be calculated to be $g_{0}(0)=2 \pi \times 3.8 \mathrm{GHz}$ at this location, and $g_{0}\left(d_{\mathrm{twzr}}\right)=2 \pi \times 100 \mathrm{MHz}$ at the chosen location of the atom $d_{\mathrm{twzr}}=350 \mathrm{~nm}$, as explained in Sec. V. The single-atom cooperativity defined here as $C_{0}=4 g_{0}^{2} / \kappa \Gamma$ between a $\mathrm{Yb}$ atom and the silicon photonic crystal cavity can be estimated using the cavity linewidth $\kappa=\kappa_{i}+\kappa_{e}$ and the atomic linewidth $\Gamma=2 \pi \times$ $0.32 \mathrm{MHz}$. This corresponds to a cooperativity for an atom located at $d_{\mathrm{twzr}}$ of $C_{0}=47$. The Purcell-enhanced emission rate $\Gamma_{1 \mathrm{D}}$ is given by the Purcell factor $P=C_{0}$ and the atomic decay rate $\Gamma$ as $\Gamma_{1 \mathrm{D}}=P \Gamma$, which for this system gives $\Gamma_{1 \mathrm{D}} / 2 \pi=15 \mathrm{MHz}$.

The probability of spontaneously emitting a photon into the cavity mode $P_{\text {cavity }}$ is given by $C_{0} /\left(C_{0}+1\right)$, which is 0.98 . Thus, the total efficiency of extracting the photon from the atom into the waveguide mode is given by $\eta_{\text {ext }}=P_{\text {cavity }} \eta_{\text {coll }}$, which is 0.87 . We design the photonic cavity to taper to a nanobeam waveguide, which can then be coupled to an optical fiber using a microlens or adiabatic coupler. Efficiencies for the latter are $\eta_{\mathrm{AC}} \approx 0.95$ [72]. These parameters result in a total system efficiency of approximately 0.80 .

\section{TRAPPING A SINGLE YB ATOM NEAR A PHOTONIC CRYSTAL}

We now show that an $\mathrm{Yb}$ atom can be trapped close to the photonic crystal using only a tightly focused optical tweezer.

\section{A. Analysis of the optical tweezer trap}

Single-atom detection and addressing of alkalineearth(-like) atoms is a growing area of research interest. 
Quantum gas microscopy of $\mathrm{Yb}$ has been demonstrated [73], and large two-dimensional tweezer arrays of $\mathrm{Yb}$ [74] and strontium (Sr) $[75,76]$ have recently been reported. Further, cooling of single alkaline-earth(-like) atoms close to the motional ground state of an optical tweezer has recently been demonstrated for $\mathrm{Sr}[75,76]$, and cooling of alkali atoms optically trapped approximately 300 $\mathrm{nm}$ from a room-temperature surface has recently been observed [77].

For a tweezer wavelength of $\lambda_{\text {twzr }} \approx 473 \mathrm{~nm}$ (depending on the tweezer polarization [75]), there is a "magic" wavelength for which the polarizability of ${ }^{1} S_{0}$ and ${ }^{3} P_{1}$ are identical $[78,79]$. This is particularly useful for cooling the atom in the tweezer $[75,76]$. Coincidentally, the polarizability of ${ }^{3} P_{0}$ is also similar [80]. Moreover, the polarizability at this wavelength is large, which allows deep traps to further mitigate surface forces. As such, we propose to use the approximately $473-\mathrm{nm}$ wavelength for generating tightly focused optical tweezers, although easily accessable wavelengths such as $532 \mathrm{~nm}$ are an alternative as they have been used in a similar magic configuration $[73,74]$.

In order to further understand the design constraints and tweezer trap properties, we describe here the polarizability at the trapping wavelength $\lambda_{\text {twzr }}$ and the tweezer waist that can be generated with numerical aperture NA $\approx 0.7 \mathrm{objec}-$ tive [73]. The polarizability of ${ }^{1} S_{0}$ and ${ }^{3} P_{1}$ at $\lambda_{\text {twzr }}$ is $\alpha=$ $-18 \mathrm{~Hz} /\left(\mathrm{W} / \mathrm{cm}^{2}\right)$ [80]. For an objective of $\mathrm{NA} \approx 0.70$, the $1 / e^{2}$ waist radius of a tweezer that can be generated with this wavelength is $w_{\text {twzr }} \approx 330 \mathrm{~nm}$ and the corresponding Rayleigh range is $R_{\mathrm{twzr}} \approx 730 \mathrm{~nm}$. An optical power of $1.0 \mathrm{~mW}$ is required for a trap depth of $U_{\mathrm{twzr}} \approx 0.5$ $\mathrm{mK}$, and the trapping frequencies are $\omega_{R} \approx 2 \pi \times 150 \mathrm{kHz}$ and $\omega_{z} \approx 2 \pi \times 48 \mathrm{kHz}$. The polarizability of the ${ }^{3} D_{1}$ state is not well known, but it is only populated during a $\pi$ pulse for photon-spin entanglement and during readout (see Appendix B). To mitigate deleterious effects from a polarizability mismatch on the ${ }^{3} P_{0} \leftrightarrow{ }^{3} D_{1}$ transition, we propose to switch the trap off during excitation [19]. Atom survival probability in the absence of a trap is known to be high for times of several microseconds [81], which is much longer than the Purcell-enhanced emission timescale $\Gamma_{1 \mathrm{D}}^{-1}=11 \mathrm{~ns}$.

The temperature of a typical $\mathrm{Yb}$ magneto-optical trap (MOT) operating on the ${ }^{1} S_{0} \leftrightarrow{ }^{3} P_{1}$ transition is < $10 \mu \mathrm{K}[73,80]$. This temperature would correspond to $n_{R}=0-1$ motional quanta in the radial direction and $n_{z}=2-3$ motional quanta in the axial direction, and further cooling in a tweezer has been demonstrated with Sr $[75,76]$. Such conditions lead to thermal $1 / e^{2}$ atomic wave-function radii of $\sigma_{R} \approx 30 \mathrm{~nm}$ in the radial direction and $\sigma_{z} \approx 80 \mathrm{~nm}$ in the axial direction. The circles in Fig. 4(d) are meant to roughly represent the size of the atomic wave function and illustrate how small it is compared to the mode profile of the cavity field. Note that the nodal spacing of the cavity is larger here compared to previous work [18] because of the longer wavelength and, thus, the evanescent field coupling is expected to be more homogeneous over the tweezer trap volume.

\section{B. Atom trapping and imaging near the photonic crystal}

We consider a tweezer focused at a distance $d_{\mathrm{twzr}}=$ $350 \mathrm{~nm}$ from the photonic crystal, as shown in Figs. 5(a) and 5(b). This distance was chosen to be slightly larger than the waist of the tweezer $w_{\mathrm{twzr}} \approx 330 \mathrm{~nm}$ to minimize the impact of scattered fields from the photonic crystal. Further, we propose to use the chip geometry shown in Fig. 5(c) and discussed in the next subsection, which allows the tweezer to be translated with respect to the cavities on the chip and the MOT [18] using an acousto-optic deflector or spatial light modulator. This is advantageous since atomic flux on the device is known to have detrimental effects on photonic structures $[82,83]$.

A significant difference between alkali and alkalineearth(-like) atoms is the size and temperature of their MOTs. For alkalis, typically only one atomic transition is

(a)

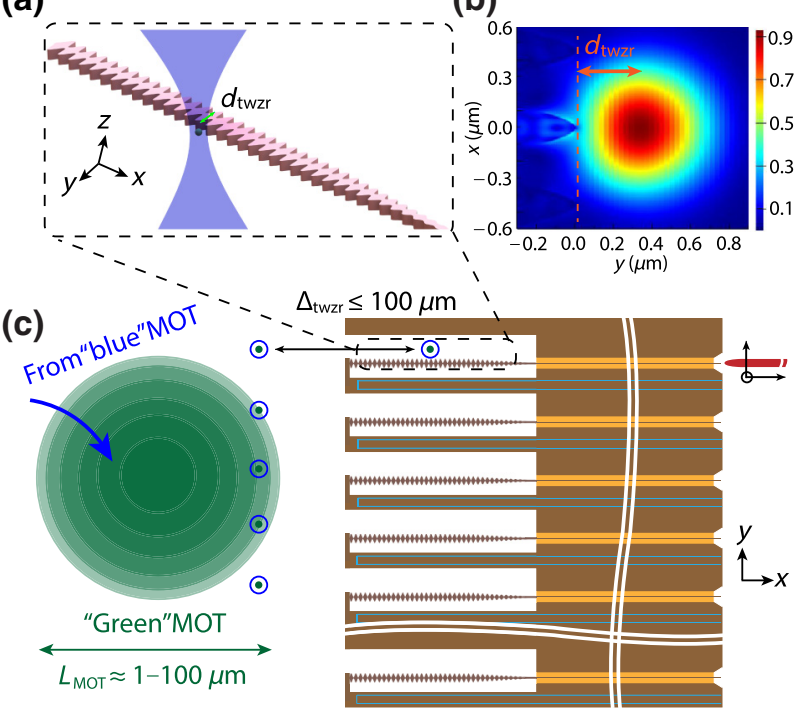

FIG. 5. Coupling an $\mathrm{Yb}$ atom to a silicon photonic crystal cavity via an optical tweezer trap. (a) An enlargement illustrating the atom in a tweezer near the device at a distance $d_{\text {twzr }}$. (b) The electic field magnitude of the tweezer trap in arbitrary units at a distance of $d_{\mathrm{twzr}}=350 \mathrm{~nm}$ from the edge of the device. (c) Schematic of the silicon chip. The silicon top layer is brown and the insulator layer below is orange. The blue microstrips on the chip are used for Ohmic temperature control and applying rf magnetic fields. The lensed fiber on the right can be coupled to any cavity using a three-dimensional (3D) translation stage. Wavy white lines indicate cuts to show the entire chip. The green circle on the left is the $\mathrm{Yb}$ MOT. Atoms can be shuffled between it and the device using optical tweezers. This architecture allows simultaneous operation of multiple $\mathrm{Yb}$-cavity nodes on a single device. 
used for laser cooling and optical molasses. Conversely, alkaline-earth(-like) atoms are typically laser cooled in two stages: the broad ${ }^{1} S_{0} \leftrightarrow{ }^{1} P_{1}$ transition for initial loading and the narrow ${ }^{1} S_{0} \leftrightarrow{ }^{3} P_{1}$ transition for cooling to $<10 \mu \mathrm{K}[73,80]$ as mentioned above. Further, the narrow linewidth of the cooling transition in the second stage allows for flexibility in the MOT size and position by adjusting the magnetic field gradient and offset. Hence, a small and cold second-stage "green" MOT could be moved to within approximately $100 \mu \mathrm{m}$ of the silicon chip using magnetic fields, which is within the field of view of typical microscope objectives. This approach enables easy tweezer transport with acousto-optic deflectors [45] (see Fig. 5) and does not require translation of the objective. The large first-stage "blue" MOT could be millimeters from the chip at all times. This technique reduces atomic flux onto the device compared to previous work $[17,18]$ and also mitigates the detrimental effect of the photonic structure on the MOT.

Surface effects on the atom are substantially mitigated in our approach compared to previous work [18]. Operating at $350 \mathrm{~nm}$ from the surface instead of $200 \mathrm{~nm}$ decreases the surface potential to approximately $13 \%$ and surface force to approximately $7 \%$ of that of the previous work [18] (see the Supplementary Material in Ref. [18] and Ref. [84] for electric dipole polarizabilities at imaginary frequencies used in this calculation). In addition, the external corregation used in our photonic crystal design further reduces the effective surface area interacting with the atom where only the surface areas at the antinodes [see Figs. 1 and 5(b)] contribute significantly to the surface force. We show the qualitative scaling of the surface force with distance in Fig. 4(e), but we leave a quantitative assessment for further study. We can compare the surface force with the dipole force in the tweezer potential. We expect the surface force at $d_{\mathrm{twzr}}$ to be approximately $100 \mathrm{kHz} / \mu \mathrm{m}$ or less, while the maximum dipole force for a tweezer as described above with $1-\mathrm{mK}$ depth is approximately $50 \mathrm{MHz} / \mu \mathrm{m}$, and the use of deeper traps is possible.

Now, we consider absorption of the tweezer light by the silicon nanocavity. Absorption can have two deleterious effects: it can cause heating of the structure that will alter the cavity properties, and it can generate free carriers that increase the optical absorption in the telecom band $[85,86]$. The absorption coefficient of silicon at a wavelength of approximately $470 \mathrm{~nm}$ is $\alpha_{\mathrm{Si}} \approx 2 \times 10^{4} \mathrm{~cm}^{-1}$ [87], which means that approximately $18 \%$ of incident tweezer light will be absorbed by our cavity of $100-\mathrm{nm}$ thickness. For a $1-\mathrm{mW}$ tweezer of waist $w_{\mathrm{twzr}}=330 \mathrm{~nm}$ at a distance from the surface of $d_{\mathrm{twzr}}=350 \mathrm{~nm}$, we estimate that $<3 \mu \mathrm{W}$ is incident on the device and, thus, $<530 \mathrm{nW}$ is absorbed. Given the thermal conductivity of silicon and the dimensions of the proposed nanocavity, we expect an approximately $0.3-\mathrm{K}$ temperature difference in the vicinity of the tweezer relative to the chip, which serves as a thermal reservoir. The temperature dependence of the index of refraction causes a shift in the cavity resonance frequency at the $10^{-5} / \mathrm{K}$ level [85]. Hence, we expect an approximately $3 \times 10^{-6}$ fractional shift in the cavity resonance, which is much less than $1 / Q_{e}>10^{-5}$ for the cavity.

To mitigate the effect of free-carrier generation in the silicon nanocavity, we propose to switch the tweezer off during the telecom-photon emission phases, such as spinphoton entanglement and readout. The weak tweezer illumination onto the device causes free electron-hole pairs that decay within tens of nanoseconds $[85,86]$. This is fast compared to the allowed free expansion time of the atom with high-probability retrapping (several microseconds), so waiting for hundreds of nanoseconds to ensure electron-hole pair decay is feasible. Since the recoil energy of a telecom photon is low, we expect heating of the atom during these pulses to be sufficiently small.

Coherent scattering from the nanocavity must also be considered because it could alter the trapping potential. We calculate the trapping potential in the presence of scattering from the silicon device using a finite-difference time-domain simulation. We simulate a Gaussian beam with a waist of $330 \mathrm{~nm}$ focused at a distance of $d_{\mathrm{twzr}}=350$ $\mathrm{nm}$ from the edge of the photonic crystal cavity. The results are shown in Fig. 5(b), where the magnitude of the electric field is shown on a relative scale. The trap perturbations are below $\left|E_{\mathrm{twzr}}\right| \approx 0.2\left(I_{\mathrm{twzr}} \approx 0.1\right)$ and they occur only at distances of $\geq d_{\mathrm{twzr}}$ from the center of the tweezer. This effect is negligible, particularly for a cold atom.

The ability to have many photonic cavities per chip facilitates overcoming fabrication error and device degradation, but also opens up the possibility to couple an array of atoms in optical tweezers [45] to an array of cavities. This would enable multiqubit repeater nodes as discussed in Secs. II and III and Appendix B. An array of cavities can be fabricated such that their separation is as small as approximately $10 \mu \mathrm{m}$ [as in Fig. 5(c)]. The cavities are coupled to a microlens coupler or adiabatic coupler and the fiber can be aligned and coupled to any individual cavity on the chip using a three-axis stage, as shown in Fig. 5(c). Each cavity is independently temperature controlled with its own tungsten heating strip [light blue lines in Fig. 5(c)] and, thus, each cavity in the array can be individually tuned into resonance with the atomic transition [88]. The tungsten strip is also used for applying rf pulses to control the nuclear spin (Appendix B).

\section{CONCLUSION AND OUTLOOK}

We have illustrated that silicon-based photonics combined with tweezer-trapped ${ }^{171} \mathrm{Yb}$ atoms are a candidate system for quantum optics in the telecom band. The strong 1.4- $\mu \mathrm{m}$ transition from the ${ }^{3} P_{0}$ "clock" state of $\mathrm{Yb}$ enables $15-\mathrm{MHz}-$ bandwidth emission when coupled to a silicon photonic cavity and the nuclear spin allows for a 
coherent quantum memory. Further, we propose a simple and robust trapping protocol that enables atoms to be coupled to silicon nanophotonics and even facilitates an array of atoms coupled to an array of cavities. Furthermore, we illustrate the potential of our platform for quantum repeaters.

Moreover, there are several other applications of this system that include quantum photonic circuits, novel platforms for long-range interactions, and many-body physics [46]. Our system may enable the direct integration of neutral-atom quantum computers into a quantum network, in which quantum gates can be performed using local exchange [50,51] or Rydberg [52] interactions. Further, this system opens up the possibility to implement an optical clock network [89] by using the ${ }^{1} S_{0} \leftrightarrow{ }^{3} P_{0}$ optical clock qubit [53].

Alternative cavity designs based on free-space optics could offer different possibilities. We consider a fiber Fabry-Perot cavity in Appendix C. Finally, we note that a similar telecom-wavelength atomic system could be created with $\mathrm{Yb}^{+}$ions, where a group of transitions from a metastable state has convenient wavelengths of 1450 and $1650 \mathrm{~nm}$. We analyze a $\mathrm{Yb}^{+}$ion coupled with a fiber Fabry-Perot cavity in Appendix D, but we note that ion trapping near dielectric materials poses other technical challenges.

\section{ACKNOWLEDGMENTS}

We acknowledge Mohammad Mirhosseini, Alexandre Cooper-Roy, and Matthew D. Shaw for useful discussions and Hengjiang (Jared) Ren with help with COMSOL simulations. We also ackowledge Hannes Bernien and Jeff Thompson for critical reading of the manuscript. J.P.C. acknowledges support from the Caltech PMA Division for postdoctoral fellowship funding, A.S. acknowledges support from the Caltech IQIM for postdoctoral fellowship funding, and N.S. acknowledges funding by the Alliance for Quantum Technologies' Intelligent Quantum Networks and Technologies (INQNET) research program. We acknowledge funding provided by the Institute for Quantum Information and Matter, a NSF Physics Frontiers Center (NSF Grant No. PHY-1733907). This work is also supported by the NSF CAREER award, the Sloan Foundation, and by the NASA/JPL President's and Director's Fund.

\section{APPENDIX A: DIPOLE MATRIX ELEMENTS AND POLARIZATION CONSIDERATIONS}

In order to quantify the coupling to a cavity, we must calculate the dipole matrix element of the desired atomic transition: ${ }^{3} P_{0}\left|F=1 / 2, m_{F}=1 / 2\right\rangle \leftrightarrow{ }^{3} D_{1} \mid F=3 / 2, m_{F}=$ $3 / 2\rangle$ (see Appendix B for a detailed quantum repeater scheme). The ${ }^{3} P_{0} \leftrightarrow{ }^{3} D_{1}$ transition has been carefully measured because of its relevance to $\mathrm{Yb}$ clock precision
[78]. Given that $\Gamma^{3} D_{1} \rightarrow^{3} P_{0}=2 \times 10^{6} \mathrm{~s}^{-1}$, we arrive at $\mu=$ $\left\langle F=1 / 2, m_{F}=1 / 2\left|e \hat{\mathbf{r}}_{q}\right| F^{\prime}=3 / 2, m_{F^{\prime}}=3 / 2\right\rangle=1.38 \times$ $10^{-29} \mathrm{C} \mathrm{m}$, or $1.63 \mathrm{a}_{0} \mathrm{e}$, in which the polarization is taken to be $q=+1\left(\sigma^{+}\right)$[90]. However, purely circular polarization cannot be supported by the modes of the photonic structure (see Sec. IV), and so the effective dipole matrix element is reduced by $\sqrt{2}$ upon decomposing $\sigma^{+}$ into a combination of linear polarizations. The "quantization axis" is assumed to be determined by the electric field of the optical tweezer $E_{\text {twzr }}$ (see Sec. IV) and the external magnetic field $B_{\text {ext }}$ (see Appendix B) is assumed to be parallel. To drive the $\sigma^{+}$transition, we require the electric field of the mode in the cavity $E_{\text {cavity }}$ to be perpendicular to the quantization field axis $E_{\text {cavity }} \perp E_{\mathrm{twzr}}, B_{\text {ext }}$.

Finally, we study the emission branching ratio from ${ }^{3} D_{1}$ to ${ }^{3} P_{J}$, for $J=0,1,2$ as shown in Fig. 2. On the basis of fine structure alone, the branching ratio from ${ }^{3} D_{1}$ to ${ }^{3} P_{0},{ }^{3} P_{1}$, and ${ }^{3} P_{2}$ is $60: 3: 1$, respectively. Therefore, in the absence of Purcell enhancement, the probability of emitting the desired photon from ${ }^{3} D_{1}$ is $93.3 \%$. The Purcell enhancement for the decay to ${ }^{3} P_{0}$ based on our analysis is 47 and the corresponding branching ratio becomes 2820:3:1. Hence, the probability of emitting the desired photon with Purcell enhancement is $99.9 \%$. However, when the hyperfine structure is included in this analysis and decay from the specific state of interest ${ }^{3} D_{1} \mid F=$ $\left.3 / 2, m_{F}=3 / 2\right\rangle$ is considered, a more detailed calculation is required [90], upon which we find the following values. In the absence of Purcell enhancement, the branching ratio is 20.9:1.9:1, for which the probability of emitting the desired photon is $88 \%$. With Purcell enhancement, the branching ratio is $982: 1.9: 1$, for which the probability of emitting the desired photon is $99.7 \%$.

\section{APPENDIX B: QUANTUM REPEATER IMPLEMENTATION}

In this section, we describe a quantum repeater based on a network of ${ }^{171} \mathrm{Yb}$ atoms coupled to photonic crystal cavities. The repeater involves dividing a long channel of length $L$ into $2^{n}$ elementary links of length $L_{0}$ that are connected by nodes that feature a pair of trapped atoms. The integer $n$ is often referred to as the number of nesting levels [62]. Atom-atom entanglement that spans $L$ is achieved by entangling atoms that are separated by $L_{0}$ and by performing entanglement swapping between atoms that are located at each node. Figure 3(b) depicts two elementary links and entanglement swapping at one node. We consider the scheme of Barrett and Kok [54,55] for the generation of remote spin-spin entanglement and then calculate the rate of distribution of a Bell state.

\section{Level scheme and protocol}

We consider the three levels of ${ }^{171} \mathrm{Yb}$ shown in Fig. 3(a) using solid colors. These consist of an excited $m_{F}=3 / 2$ 
Zeeman level of the ${ }^{3} D_{1}$ manifold and a pair of $m_{F}=$ $\pm 1 / 2$ nuclear spin levels of the ${ }^{3} P_{0}$ manifold that form the ground level. The ${ }^{3} P_{0}$ state can be populated from the ${ }^{1} S_{0}$ ground state by using the "clock" transition [53] or by multiphoton processes [91]. The $m_{F}=3 / 2$ and $m_{F}=1 / 2$ Zeeman levels of the ${ }^{3} D_{1}$ level are separated by 0.47 $\mathrm{MHz} / \mathrm{G}$, which is much larger than the $752 \mathrm{~Hz} / \mathrm{G}$ splitting of the $m_{F}= \pm 1 / 2$ levels of ${ }^{3} P_{0}$.

The first step of the repeater is to generate spin-photon entanglement and then, using two-photon detection, spinspin entanglement between atoms that are separated by one elementary link. We begin by preparing each atom in an equal superposition of the $m_{F}= \pm 1 / 2$ nuclear states of the ${ }^{3} P_{0}$ ground level: $1 / \sqrt{2}(|\uparrow\rangle+|\downarrow\rangle)$. This is accomplished by a rf field despite the relatively small gyromagnetic ratio of the spin $\gamma_{N} / 2 \pi=752 \mathrm{~Hz} / \mathrm{G}$. Nonetheless, this favorably results in a weak coupling of the spin to the environment $[92,93]$. We propose to split these states with a magnetic field of $B_{\text {ext }}=200 \mathrm{G}$. With the fabricated microstripline resonator [94] described in Sec. V, we expect Rabi frequencies of tens of kilohertz using tens of watts of $\mathrm{rf}$ power. This allows $\pi$ pulses to be performed on timescales that are much less than the time to establish entanglement over an elementary link (see Appendix 2).

Next, each atom is excited by a short laser pulse that is resonant with the $|\uparrow\rangle \rightarrow|e\rangle$ transition, as indicated by $\lambda_{\text {cavity }}$ in Fig. 3(a). As described in Sec. V, this transition is strongly coupled to the cavity and, thus, the resultant spontaneous emission locally entangles the spin and photon number in the Bell state $1 / \sqrt{2}(|\uparrow, 1\rangle+|\downarrow, 0\rangle)$, in which 1(0) represents the presence (absence) of an emitted photon.

The photons that are emitted by each atom are directed to a beam splitter which is located halfway between nodes; see Fig. 3(b). If the photons that are emitted by each atom are indistinguishable, detection of one photon after the beam splitter heralds spin-spin entanglement or, because of potential loss and imperfections, a spin-spin product state between each atom $[54,55]$. To avoid the latter, a $\pi$ pulse inverts the $m_{F}= \pm 1 / 2$ spins and the transition is optically excited a second time. The detection of a photon in both rounds heralds the creation of a spin-spin Bell state between each atom $1 / \sqrt{2}\left(|\uparrow, \downarrow\rangle_{A B} \pm|\downarrow, \uparrow\rangle_{A B}\right)$, in which the relative phase is defined according to whether both photons are detected on the same or different output ports of the beam splitter.

Entanglement swapping is accomplished by performing a similar procedure as to generate heralded entanglement —optical excitation, single-photon detection, spin flip, and detection of a second photon. This procedure limits the swapping efficiency to at most 50\% [95]. A deterministic swapping process, which allows improved scaling, could be achieved by trapping two atoms using two tweezers within a single cavity and exploiting photonmediated deterministic intracavity gates [46].
A limitation of our entanglement generation process is the isolation of the $|\uparrow\rangle \rightarrow|e\rangle$ transition relative to the other transitions in the ${ }^{3} D_{1}$ state [see Fig. 3(a)]. The splitting between the $m_{F}=3 / 2$ and $m_{F}=1 / 2$ states is 470 $\mathrm{kHz} / \mathrm{G}$ and this must be compared to the Purcell-enhanced linewidth $\Gamma_{1 \mathrm{D}}$. We choose a field of $B_{\text {ext }}=200 \mathrm{G}$ for which $\Delta=2 \pi \times 93 \mathrm{MHz}$. For $\Gamma_{1 \mathrm{D}}=2 \pi \times 15 \mathrm{MHz}$ and Rabi frequency $\Omega=\Gamma_{1 \mathrm{D}}$, the off-resonant scattering rate is $\Gamma_{\mathrm{SC}}=2 \pi \times 600 \mathrm{kHz}$. The readout fidelity is assumed to be $\mathcal{F}_{\mathrm{RO}}=1-\Gamma_{\mathrm{SC}} / \Gamma_{1 \mathrm{D}}$, which is $>0.99$. Note that this value is even slightly improved when considering the Clebsch-Gordan coefficients for the different pathways.

\section{Entanglement distribution rate}

We quantify the distribution rate of a Bell state using our repeater scheme, showing that it outperforms an approach based on the direct transmission of photons. We denote the success probability for an atom to emit a photon into a single mode fiber (e.g., system efficiency) to be $p$, which includes the probability to prepare the initial state, the spontaneous emission of a photon into the cavity mode, and the coupling into a fiber. The probability of the twophoton measurement at the center of the elementary link is given by $P_{0}=\frac{1}{2} p^{2} \eta_{t}^{2} \eta_{d}^{2}$ in which $\eta_{t}=e^{-L_{0} /\left(2 L_{\text {att }}\right)}$ is the fiber transmission with attenuation length $L_{\text {att }}=12 \mathrm{~km}$. This corresponds to losses of $0.35 \mathrm{~dB} / \mathrm{km}$ at $1.4 \mu \mathrm{m}$ using a hydrogen-aged single mode fiber, which has been recently deployed for modern infrastructure (see, e.g., SMF-28e for ITU-T G.562D standards, manufactured by Corning, Inc. [96]).

The spin-spin entanglement creation step is repeated at time intervals of the communication time $L_{0} / c$, in which $c=2 \times 10^{8} \mathrm{~m} / \mathrm{s}$ is the speed of light in fiber. Thus, the average time to produce entanglement that spans an elementary link is $T_{L_{0}}=\left(L_{0} / c\right)\left(1 / P_{0}\right)$. Using the beamsplitter approach depicted in Fig. 3(b), the efficiency of the entanglement swapping operation is $P_{s}=\frac{1}{2} p^{2} \eta_{d}^{2}$, while a deterministic gate allows $P_{s}=1$, assuming the gate fidelity is unity. Therefore, the total time for the distribution of an entangled pair over distance $2 L_{0}$ is given by $T_{2 L_{0}}=\frac{3}{2}\left(L_{0} / c\right)\left(1 / P_{0} P_{s}\right)$ and the average time to distribute an entangled pair over distance $L$ is $T_{L} \approx$ $\left(\frac{3}{2}\right)^{n-1}\left(L_{0} / c\right)\left(1 / P_{0}\right)\left(P_{s}\right)^{n}$. The factor of $3 / 2$ arises because entanglement has to be created over two links before the swapping is performed [62,97].

For the discussion in Sec. III, we assume $p=0.8$, which is given by the $\eta_{\text {ext }} \eta_{\text {taper }} \eta_{\text {AC }}$ estimated in Sec. IV, a detection efficiency of 0.9 , and that the lifetime of the ${ }^{3} P_{0}$ level is much longer than the distribution time. This detection efficiency is straightforwardly achieved using superconducting nanowires, which have been demonstrated at $1.5 \mu \mathrm{m}$ [98]. Note that the spontaneous Purcell-enhanced emission time from ${ }^{3} D_{1}$ is $1 / \Gamma_{1 \mathrm{D}} \approx 11 \mathrm{~ns}$, which is negligible compared to the time to distribute entanglement 
over an elementary link. Figures 3(c) and 3(d) show the repeater performance based on the above analysis, plotted versus total distance for $2^{4}$ nodes [Fig. 3(c)] and versus the number of nodes for a total distance of $600 \mathrm{~km}$ [Fig. 3(d)].

In our analysis, we assume that the emission bandwidths of each $\mathrm{Yb}$ atom-cavity system are identical. However, variations in the cavity properties and tweezer-cavity distance result in a variation in the Purcell-enhanced decay rate. For our proposed remote entanglement generation scheme, this variation causes a reduction in the entanglement generation efficiency, but does not impact the entanglement fidelity. Compensation for this variation could be achieved by applying a standard frequency, e.g., fiber-Bragg grating, or temporal filter to the two-photon coincidence signal $[99,100]$.

\section{APPENDIX C: COUPLING YB TO A FIBER GAP FABRY-PEROT CAVITY}

In this section, we discuss an alternative approach to efficiently interface a single atom with a single telecom photon in a fiber using a fiber-based FabryPerot (FP) resonator [101-103]. While fiber-based FabryPerot resonators have significantly larger mode volumes (approximately $1000 \lambda^{3}$ ) that result in reduced vacuum Rabi frequencies, we show that they can achieve high enough cooperativities necessary to extract single photons directly into a telecom fiber with sufficiently high efficiency.

We begin by considering the geometry of fiber FP cavities, as shown in Fig. 6(a). Typical heights $H$ of the cavity claddings are $125 \mu \mathrm{m}$, so in order to focus a tweezer and image an atom inside with high NA, we assume a cavity length $L=150 \mu \mathrm{m}$. We choose radii of curvature $R$ of the cavity mirrors to be $R=100 \mu \mathrm{m}$, which is a typical value for such $\mathrm{CO}_{2}$-laser etching techniques [103].
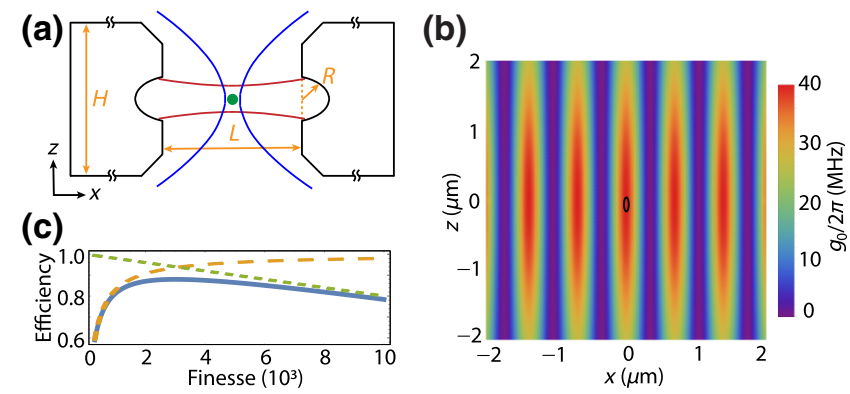

FIG. 6. Tweezer trapping in a fiber Fabry-Perot cavity. (a) The geometry of the fiber FP cavity and the tweezer trap at the center. (b) An enlargement of the center $\pm 2 \mu \mathrm{m}$ of the cavity mode. The black ellipse shows a liberal estimate of the atomic wave function in a tweezer trap as described in Sec. V. (c) The efficiencies associated with the fiber cavity vs $F_{e}$ (see text). $P_{\text {cavity }}$ (orange long-dashed line), $\eta_{\text {coll }}$ (green short-dashed), and their product $\eta_{\text {ext }}$ (blue).
This geometry at a wavelength of $\lambda=1388.8 \mathrm{~nm}$ gives a mode waist of $\omega_{0}=4.4 \mu \mathrm{m}$ and a mode volume of $V_{m}=2.3 \times 10^{-15} \mathrm{~m}^{3}$, or $842 \lambda^{3}$.

We also analyze the mode profile in this cavity in a similar way to the photonic cavity and an enlargement of the central $\pm 2 \mu \mathrm{m}$ is shown in Fig. 6(b). The black ellipse shows a liberal estimate of the atomic wave function in a tweezer as described in Sec. V. The only requirement in this system is that the atom is centered on the antinode as shown in Fig. 6(b). Noting that the $y$ axis into the page has the same profile as the $z$ direction, this system requires an overall less precise alignment than the photonic cavity.

The coherent coupling rate $g_{0}$ between the cavity and the atom is given by [103]

$$
g_{0}=\sqrt{\frac{\mu^{2} \omega}{2 \hbar \epsilon_{0} V_{m}}},
$$

where $\mu$ is the dipole matrix element, $\omega$ is the angular frequency of the cavity and the atomic transition, and $\epsilon_{0}$ is the permittivity of free space. For this geometry and the value of $\mu$ discussed above, we obtain a coherent coupling rate of $g_{0}=2 \pi \times 39 \mathrm{MHz}$. Note that here the cavity supports two degenerate polarizations, so the cavity field can have perfect polarization overlap with the transition dipole. As with the photonic crystal cavity, we design the fiber cavity such that one mirror has lower reflection and allows for coupling photons into and out of the cavity $[48,70,71]$. Now, we choose a finesse of $F_{e}=2000$, which is well below maximum finesse values of $>10^{5}$ [103]. The cavity linewidth $\kappa_{e}=\pi c /(L F)$ for these values is $\kappa=2 \pi \times 500 \mathrm{MHz}$, and the free spectral range FSR $=2 \pi c /(2 L)=2 \pi \times 1.0$ THz.

We can now estimate the single-atom cooperativity [103], defined here for consistency with the photonic crystal as $C_{0}=4 g_{0}^{2} / \kappa \Gamma$. For our values, we arrive at $C_{0}=$ 39 , for which the probability of emission of a spontaneous photon into the cavity mode is $P_{\text {cavity }}=C_{0} /\left(C_{0}+1\right)=$ 0.97. We assume an intrinsic finesse of $F_{i}=5 \times 10^{4}$ [70, $103]$, for which we can again define a collection efficiency $\eta_{\text {coll }}$ of extracting the photon into the waveguide mode, which is given by $\eta_{\text {coll }}=1-F_{e} / F_{i}$. For $F_{e}=2000$, this corresponds to $\eta_{\text {coll }}=0.96$. The efficiency of extracting a

TABLE II. The values relevant for a quantum repeater with a fiber FP cavity and the sections of the text in which they are described.

\begin{tabular}{lcc}
\hline \hline Parameter & Section & Value \\
\hline Bandwidth & Appendix C & $2 \pi \times 13 \mathrm{MHz}$ \\
Wavelength & II & $1.39 \mu \mathrm{m}(0.35 \mathrm{~dB} / \mathrm{km})$ \\
System efficiency & Appendix C & 0.80 \\
Memory & II, Appendix B & $\leq 26 \mathrm{~s}$ \\
Readout fidelity & Appendix C & $>0.99$ \\
\hline \hline
\end{tabular}


(a)

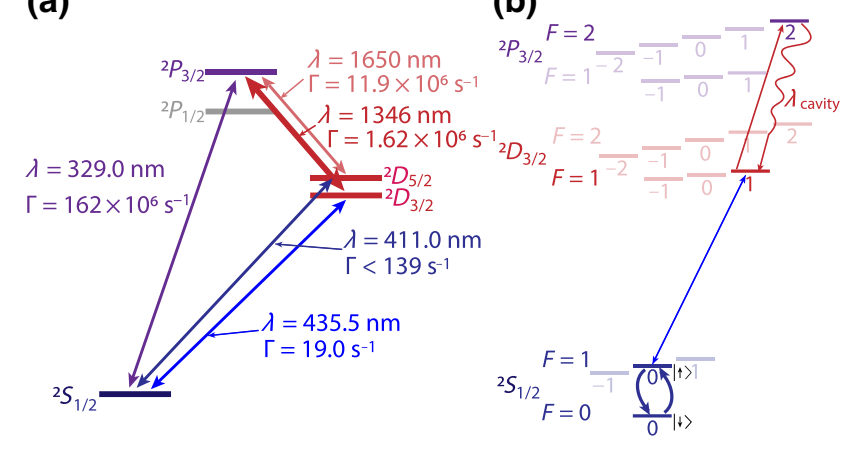

FIG. 7. $\mathrm{Yb}^{+}$level structure, and excitation scheme for ${ }^{171} \mathrm{Yb}^{+}$. (a) The relavent levels of $\mathrm{Yb}^{+}$, showing their wavelengths and linewidths. The thick red line shows the proposed telecom transition. (b) Three-level scheme by which quantum information in the ground state can be entangled with a telecom photon via the proposed telecom transition resonant with the cavity mode.

photon from the atom into the waveguide mode is given by the product of these $\eta_{\text {ext }}=P_{\text {cavity }} \eta_{\text {coll }}$, which is 0.94 . $\eta_{\text {ext }}$ is maximized for $F_{e}=2000$, as shown in Fig. 6(c).

However, another factor emerges because we require the waveguide mode of the optical fiber to be single mode (SM). This is necessary since indistinguishable photons are required for Bell state measurements. Representative efficiencies for coupling a photon in a cavity similar to our design into a SM fiber are $\eta_{\mathrm{SM}} \lesssim 0.85$ [103]. The photon acquisition efficiency is thus $\eta_{\mathrm{PA}}=\eta_{\mathrm{ext}} \eta_{\mathrm{SM}}=0.80$.

As with the photonic crystal in the main text, we show a table for the fiber FP cavity of the quantities relevant for a quantum repeater in Table II. The Purcell-enhanced linewidth is given by $\Gamma_{1 \mathrm{D}}=(1+P) \Gamma$, where $P=C_{0}$. For the fiber FP cavity, $\Gamma_{1 \mathrm{D}}=2 \pi \times 13 \mathrm{MHz}$. For the same $\mathrm{B}_{\mathrm{ext}}=200 \mathrm{G}$ and for $\Omega=\Gamma_{1 \mathrm{D}}$, we get $\Gamma_{\mathrm{SC}}=2 \pi \times 390$ $\mathrm{kHz}$ and $\mathcal{F}_{\mathrm{RO}}>0.99$.

\section{APPENDIX D: TELECOM NETWORKS WITH YB ${ }^{+}$ IONS}

We now discuss the possibility of using $\mathrm{Yb}^{+}$ions in fiber FP cavities. $\mathrm{Yb}^{+}$has strong telecom transitions that remain largely unused in experiments. Yet, they have been studied carefully by theorists because $\mathrm{Yb}$ is a platform for parity violation measurements [104-108]. Similar to the scheme in neutral $\mathrm{Yb}$, the telecom transitions in $\mathrm{Yb}^{+}$ are from metastable states ${ }^{2} D_{3 / 2}$ and ${ }^{2} D_{5 / 2}$, which are strongly coupled to a higher-lying state ${ }^{2} P_{3 / 2}$ via wavelengths of 1346 and $1650 \mathrm{~nm}$, respectively. The relevent level structure is shown in Fig. 7(a). The metastable ${ }^{2} D_{3 / 2}$ and ${ }^{2} D_{5 / 2}$ states are very weakly connected to the ground state via clocklike transitions, similar to the case of neutral $\mathrm{Yb}$.

The scheme described in Fig. 7(b) is designed to include the ground-state hyperfine qubit, which has become the workhorse of quantum information processing with trapped ions. One of these qubit states can be mapped to the ${ }^{2} D_{3 / 2}$ manifold by driving the electric quadrupole (E2) clock transition [109]. An excitation from this state to the ${ }^{2} P_{3 / 2}$ allows one of the ground hyperfine qubit states to be entangled with a telecom photon that is strongly coupled to the cavity. The mapping back to the ground state is done by performing a $\pi$ pulse with the clock laser.

We choose the ${ }^{2} D_{3 / 2}$ state rather than ${ }^{2} D_{5 / 2}$ because it is easier to eliminate other decay pathways from the ${ }^{2} P_{3 / 2}$ excited state. The hyperfine splitting is $430 \mathrm{MHz}$ for ${ }^{2} D_{3 / 2}$ but only $64 \mathrm{MHz}$ for ${ }^{2} D_{5 / 2}$ [108]. This latter value is comparable to the Purcell-enhanced linewidth for the decay into the cavity mode. Note that the dipole matrix is $4.2 \mathrm{a}_{0}$-e for the transition from ${ }^{2} P_{3 / 2}$ to ${ }^{2} D_{5 / 2}$ and $1.3 \mathrm{a}_{0}$-e for the transition to ${ }^{2} D_{3 / 2}$ [107]. The Purcell-enhanced linewidth for the ${ }^{2} D_{3 / 2}$ state in a fiber FP cavity is well below the hyperfine splitting of $430 \mathrm{MHz}$.

Using the analysis outlined in Appendix A and Ref. [90], we can arrive at the exact DME for the specific scheme described in Fig. 7(b). This gives $\mathrm{DME}_{|1,1\rangle \rightarrow|2,2\rangle}=0.41 \mathrm{a}_{0^{-}}$ e. This is smaller than the case for neutral $\mathrm{Yb}$ by a factor of $>3$ even though the linewidth $\Gamma$ is similar. This is because $J>0$ and, thus, there is hyperfine splitting and more states that contribute to the total linewidth.

We believe that the fiber FP cavity considered above and in Fig. 6 is also suitable for $\mathrm{Yb}^{+}$. Indeed, a $\mathrm{Yb}^{+}$ion has already been trapped inside a fiber cavity, where the fiber length is approximately $200 \mu \mathrm{m}$ [110]. In that work, the same metastable state ${ }^{2} D_{3 / 2}$ was used, but it was coupled to an even higher excited state ${ }^{3} D[3 / 2]_{1 / 2}$ (not shown), with a transition wavelength of $935 \mathrm{~nm}$. The atomic wave function in an ion trap is of similar size to that in an optical tweezer, so the entire discussion above and in Fig. 3 applies here as well.

As for the neutral $\mathrm{Yb}$ scheme described above, this scheme for an $\mathrm{Yb}^{+}$ion could be used for creating a clock network. This transition is already used for atomic ion frequency standards [109] and so the development of a commercial technology based on this system may be more straightforward. Further, such a scheme could allow direct telecommunications between trapped-ion quantum computers to facilitate the realization of a quantum computing network [1] and it could also be used with multiple qubits at each node of the network, for which gate operations between qubits can be employed for error correction or decoherence-free subspaces [111].

[1] L.-M. Duan and C. Monroe, Colloquium: Quantum networks with trapped ions, Rev. Mod. Phys. 82, 1209 (2010).

[2] A. Reiserer and G. Rempe, Cavity-based quantum networks with single atoms and optical photons, Rev. Mod. Phys. 87, 1379 (2015). 
[3] J. I. Cirac, P. Zoller, H. J. Kimble, and H. Mabuchi, Quantum State Transfer and Entanglement Distribution Among Distant Nodes in a Quantum Network, Phys. Rev. Lett. 78, 3221 (1997).

[4] S. J. van Enk, J. I. Cirac, and P. Zoller, Photonic channels for quantum communication, Science 279, 205 (1998).

[5] H. J. Kimble, The quantum internet, Nature 453, 1023 (2008).

[6] S. Wehner, D. Elkouss, and R. Hanson, Quantum internet: A vision for the road ahead, Science 362, eaam9288 (2018).

[7] M. Uphoff, M. Brekenfeld, G. Rempe, and S. Ritter, An integrated quantum repeater at telecom wavelength with single atoms in optical fiber cavities, Appl. Phys. B 122, 46 (2016).

[8] O. Alibart, V. D'Auria, M. D. Micheli, F. Doutre, F. Kaiser, L. Labonté, T. Lunghi, É Picholle, and S. Tanzilli, Quantum photonics at telecom wavelengths based on lithium niobate waveguides, J. Opt. 18, 104001 (2016).

[9] D. E. Chang, J. S. Douglas, A. González-Tudela, C.-L. Hung, and H. J. Kimble, Colloquium: Quantum matter built from nanoscopic lattices of atoms and photons, Rev. Mod. Phys. 90, 031002 (2018).

[10] X. F. Gonzalvo, G. Corrielli, B. Albrecht, M. Grimau, M. Cristiani, and H. de Riedmatten, Quantum frequency conversion of quantum memory compatible photons to telecommunication wavelengths, Opt. Express 21, 19473 (2013).

[11] M. Bock, P. Eich, S. Kucera, M. Kreis, A. Lenhard, C. Becher, and J. Eschner, High-fidelity entanglement between a trapped ion and a telecom photon via quantum frequency conversion, Nat. Commun. 9, 1998 (2018).

[12] A. Dréau, A. Tchebotareva, A. E. Mahdaoui, C. Bonato, and R. Hanson, Quantum Frequency Conversion of Single Photons from a Nitrogen-vacancy Center in Diamond to Telecommunication Wavelengths, Phys. Rev. Appl. 9, 064031 (2018).

[13] A. Sipahigil, R. E. Evans, D. D. Sukachev, M. J. Burek, J. Borregaard, M. K. Bhaskar, C. T. Nguyen, J. L. Pacheco, H. A. Atikian, C. Meuwly, R. M. Camacho, F. Jelezko, E. Bielejec, H. Park, M. Loncar, and M. D. Lukin, An integrated diamond nanophotonics platform for quantumoptical networks, Science 354, 847 (2016).

[14] A. M. Dibos, M. Raha, C. M. Phenicie, and J. D. Thompson, Atomic Source of Single Photons in the Telecom Band, Phys. Rev. Lett. 120, 243601 (2018).

[15] K. Kutluer, M. Mazzera, and H. de Riedmatten, Solid-state Source of Nonclassical Photon Pairs with Embedded Multimode Quantum Memory, Phys. Rev. Lett. 118, 210502 (2017).

[16] T. Zhong, J. M. Kindem, J. G. Bartholomew, J. Rochman, I. Craiciu, E. Miyazono, M. Bettinelli, E. Cavalli, V. Verma, S. W. Nam, F. Marsili, M. D. Shaw, A. D. Beyer, and A. Faraon, Nanophotonic rare-earth quantum memory with optically controlled retrieval, Science 357, 1392 (2017).

[17] A. Goban, C.-L. Hung, S.-P. Yu, J. D. Hood, J. A. Munitz, J. H. Lee, M. J. Martin, A. C. McClung, K. S. Choi, D. E. Chang, O. Painter, and H. J. Kimble, Atom-light interactions in photonic crystals, Nat. Commun. 5, 3808 (2013).
[18] J. D. Thompson, T. G. Tiecke, N. P. de Leon, J. Feist, A. V. Akimov, M. Gullans, A. S. Zibrov, V. Vuletic, and M. D. Lukin, Coupling a single trapped atom to a nanoscale optical cavity, Science 340, 1202 (2013).

[19] T. G. Tiecke, J. D. Thompson, N. P. de Leon, L. R. Liu, V. Vuletic, and M. D. Lukin, Nanophotonic quantum phase switch with a single atom, Nature 508, 241 (2014).

[20] D. E. Chang, V. Vuletic, and M. D. Lukin, Quantum nonlinear optics - photon by photon, Nat. Photonics 8, 685 (2014).

[21] A. Goban, C.-L. Hung, J. D. Hood, S.-P. Yu, J. A. Muniz, O. Painter, and H. J. Kimble, Superradiance for Atoms Trapped Along a Photonic Crystal Waveguide, Phys. Rev. Lett. 115, 063601 (2015).

[22] F. Kimiaee Asadi, N. Lauk, S. Wein, N. Sinclair, C. O'Brien, and C. Simon, Quantum repeaters with individual rare-earth ions at telecommunication wavelengths, Quantum 2, 93 (2018).

[23] I. Aharonovich, D. Englund, and M. Toth, Solidstate single-photon emitters, Nat. Photonics 10, 631 (2016).

[24] D. D. Awschalom, R. Hanson, J. Wrachtrup, and B. B. Zhou, Quantum technologies with optically interfaced solid-state spins, Nat. Photonics 12, 516 (2018).

[25] E. Vetsch, D. Reitz, G. Sagué, R. Schmidt, S. T. Dawkins, and A. Rauschenbeutel, Optical Interface Created by Laser-cooled Atoms Trapped in the Evanescent Field Surrounding an Optical Nanofiber, Phys. Rev. Lett. 104, 203603 (2010).

[26] A. Goban, K. S. Choi, D. J. Alton, D. Ding, C. Lacroûte, M. Pototschnig, T. Thiele, N. P. Stern, and H. J. Kimble, Demonstration of a State-insensitive, Compensated Nanofiber Trap, Phys. Rev. Lett. 109, 033603 (2012).

[27] M. E. Kim, T.-H. Chang, B. M. Fields, C.-A. Chen, and C.L. Hung, Trapping single atoms on a nanophotonic circuit with configurable tweezer lattices, arXiv:1810.08769v1 (2018).

[28] J. M. McGuirk, D. M. Harber, J. M. Obrecht, and E. A. Cornell, Alkali-metal adsorbate polarization on conducting and insulating surfaces probed with bose-einstein condensates, Phys. Rev. A 69, 062905 (2004).

[29] D. Hunger, S. Camerer, T. W. Hänsch, D. König, J. P. Kotthaus, J. Reichel, and P. Treutlein, Resonant Coupling of a Bose-Einstein Condensate to a Micromechanical Oscillator, Phys. Rev. Lett. 104, 143002 (2010).

[30] J. M. Obrecht, R. J. Wild, and E. A. Cornell, Measuring electric fields from surface contaminants with neutral atoms, Phys. Rev. A 75, 062903 (2007).

[31] M. Antezza, L. P. Pitaevskii, and S. Stringari, Effect of the casimir-polder force on the collective oscillations of a trapped bose-einstein condensate, Phys. Rev. A 70, 053619 (2004).

[32] Y.-J. Lin, I. Teper, C. Chin, and V. Vuletic, Impact of the Casimir-polder Potential and Johnson Noise on BoseEinstein Condensate Stability Near Surfaces, Phys. Rev. Lett. 92, 050404 (2004).

[33] J. M. Obrecht, R. J. Wild, M. Antezza, L. P. Pitaevskii, S. Stringari, and E. A. Cornell, Measurement of the Temperature Dependence of the Casimir-polder Force, Phys. Rev. Lett. 98, 063201 (2007). 
[34] N. Schlosser, G. Reymond, I. Protsenko, and P. Grangier, Sub-poissonian loading of single atoms in a microscopic dipole trap, Nature 551, 485 (2017).

[35] A. M. Kaufman, B. J. Lester, and C. A. Regal, Cooling a Single Atom in an Optical Tweezer to its Quantum Ground State, Phys. Rev. X 2, 041014 (2012).

[36] J. D. Thompson, T. G. Tiecke, A. S. Zibrov, V. Vuletic, and M. D. Lukin, Coherence and Raman Sideband Cooling of a Single Atom in an Optical Tweezer, Phys. Rev. Lett. 110, 133001 (2013).

[37] V. R. Almeida, C. A. Barrios, R. R. Panepucci, and M. Lipson, All-optical control of light on a silicon chip, Nature 431, 1081 (2004).

[38] https://www.imec-int.com/en/integrated-silicon-photonics, Leuven, Belgium (https://www.imec-int.com/en/home).

[39] S. A. Miller, M. Yu, X. Ji, A. G. Griffith, J. Cardenas, A. L. Gaeta, and M. Lipson, Low-loss silicon platform for broadband mid-infrared photonics, Optica 4, 707 (2017).

[40] P. B. Dieterle, M. Kalaee, J. M. Fink, and O. Painter, Superconducting Cavity Electromechanics on a Siliconon-insulator Platform, Phys. Rev. Appl. 6, 014013 (2016).

[41] A. Pitanti, J. M. Fink, A. H. Safavi-Naeini, J. T. Hill, C. U. Lei, A. Tredicucci, and O. Painter, Strong opto-electromechanical coupling in a silicon photonic crystal cavity, Opt. Express 23, 3196 (2015).

[42] T. K. Paraïso, M. Kalaee, L. Zang, H. Pfeifer, F. Marquardt, and O. Painter, Position-squared Coupling in a Tunable Photonic Crystal Optomechanical Cavity, Phys. Rev. X 5, 041024 (2015).

[43] D. Navarro-Urrios, N. Capuj, J. Maire, M. Colombano, J. Jaramillo-Fernandez, E. Chavez-Angel, L. L. Martin, L. Mercadé, A. Griol, A. Martínez, C. M. Sotomayor-Torres, and J. Ahopelto, Nanocrystalline silicon optomechanical cavities, Opt. Express 26, 9829 (2018).

[44] D. Barredo, S. de Léséleuc, V. Lienhard, T. Lahaye, and A. Browaeys, An atom-by-atom assembler of defect-free arbitrary 2d atomic arrays, Science 354, 1021 (2016).

[45] M. Endres, H. Bernien, A. Keesling, H. Levine, E. R. Anschuetz, A. Krajenbrink, C. Senko, V. Vuletic, M. Greiner, and M. D. Lukin, Atom-by-atom assembly of defect-free one-dimensional cold atom arrays, Science 354, 1024 (2016).

[46] J. S. Douglas, H. Habibian, C.-L. Hung, A. V. Gorshkov, H. J. Kimble, and D. E. Chang, Quantum many-body models with cold atoms coupled to photonic crystals, Nat. Photonics 9, 326 (2015).

[47] J. D. Hood, A. Goban, A. Asenjo-Garcia, M. Lu, S.-P. Yu, D. E. Chang, and H. J. Kimble, Atom-atom interactions around the band edge of a photonic crystal waveguide, Proc. Natl. Acad. Sci. 113, 10507 (2016).

[48] S. Welte, B. Hacker, S. Daiss, S. Ritter, and G. Rempe, Photon-mediated Quantum Gate between Two Neutral Atoms in an Optical Cavity, Phys. Rev. X 8, 011018 (2018).

[49] A. J. Daley, M. M. Boyd, J. Ye, and P. Zoller, Quantum Computing with Alkaline-earth-metal Atoms, Phys. Rev. Lett. 101, 170504 (2008).

[50] A. J. Daley, Quantum computing and quantum simulation with group-II atoms, Quantum Inf. Process. 10, 865 (2011). Springer.
[51] A. M. Kaufman, B. J. Lester, M. Foss-Feig, M. L. Wall, A. M. Rey, and C. A. Regal, Entangling two transportable neutral atoms via local spin exchange, Nature 527, 208 (2015).

[52] M. Saffman, T. G. Walker, and K. Mlmer, Quantum information with rydberg atoms, Rev. Mod. Phys. 82, 2313 (2010).

[53] A. D. Ludlow, M. M. Boyd, J. Ye, E. Peik, and P. O. Schmidt, Optical atomic clocks, Rev. Mod. Phys. 87, 637 (2015).

[54] S. D. Barrett and P. Kok, Efficient high-fidelity quantum computation using matter qubits and linear optics, Phys. Rev. A 71, 060310 (2005).

[55] H. Bernien, B. Hensen, W. Pfaff, G. Koolstra, M. S. Blok, L. Robledo, T. H. Taminiau, M. Markham, D. J. Twitchen, L. Childress, and R. Hanson, Heralded entanglement between solid-state qubits separated by three metres, Nature 497, 86 (2013).

[56] T. Böttger, Y. Sun, C. W. Thiel, and R. L. Cone, Spectroscopy and dynamics of $\mathrm{Er}^{3+}: \mathrm{Y}_{2} \mathrm{SiO}_{5}$ at $1.5 \mu \mathrm{m}$, Phys. Rev. B 74, 075107 (2006).

[57] P. Seidler, K. Lister, U. Drechsler, J. Hofrichter, and T. Stöferle, Slotted photonic crystal nanobeam cavity with an ultrahigh quality factor-to-mode volume ratio, Opt. Express 21, 32468 (2013).

[58] T. Asano, Y. Ochi, Y. Takahashi, K. Kishimoto, and S. Noda, Photonic crystal nanocavity with a q factor exceeding eleven million, Opt. Express 25, 1769 (2017).

[59] P. C. Maurer, G. Kucsko, C. Latta, L. Jiang, N. Y. Yao, S. D. Bennett, F. Pastawski, D. Hunger, N. Chisholm, M. Markham, D. J. Twitchen, J. I. Cirac, and M. D. Lukin, Room-temperature quantum bit memory exceeding one second, Science 336, 1283 (2012).

[60] A. Reiserer, N. Kalb, M. S. Blok, K. J. M. van Bemmelen, T. H. Taminiau, R. Hanson, D. J. Twitchen, and M. Markham, Robust Quantum-network Memory using Decoherence-protected Subspaces of Nuclear Spins, Phys. Rev. X 6, 021040 (2016).

[61] S. Probst, H. Rotzinger, A. V. Ustinov, and P. A. Bushev, Microwave multimode memory with an erbium spin ensemble, Phys. Rev. B 92, 014421 (2015).

[62] N. Sangouard, C. Simon, H. de Riedmatten, and N. Gisin, Quantum repeaters based on atomic ensembles and linear optics, Rev. Mod. Phys. 83, 33 (2011).

[63] S. Pirandola, R. Laurenza, C. Ottaviani, and L. Banchi, Fundamental limits of repeaterless quantum communications, Nat. Commun. 8, 15043 (2017).

[64] R. Ikuta, T. Kobayashi, K. Matsuki, S. Miki, T. Yamashita, H. Terai, T. Yamamoto, M. Koashi, T. Mukai, and N. Imoto, Heralded single excitation of atomic ensemble via solid-state-based telecom photon detection, Optica 3, 1279 (2016).

[65] S.-P. Yu, J. D. Hood, J. A. Muniz, M. J. Martin, R. Norte, C.-L. Hung, S. M. Meenehan, J. D. Cohen, O. Painter, and H. J. Kimble, Nanowire photonic crystal waveguides for single-atom trapping and strong light-matter interactions, Appl. Phys. Lett. 104, 111103 (2014).

[66] K. Srinivasan and O. Painter, Momentum space design of high-q photonic crystal optical cavities, Opt. Express 10, 670 (2002). 
[67] J. Chan, M. Eichenfield, R. Camacho, and O. Painter, Optical and mechanical design of a "zipper" photonic crystal optomechanical cavity, Opt. Express 17, 3802 (2009).

[68] J. Chan, A. H. Safavi-Naeini, J. T. Hill, S. Meenehan, and O. Painter, Optimized optomechanical crystal cavity with acoustic radiation shield, Appl. Phys. Lett. 101, 081115 (2012).

[69] K. Fang, J. Luo, A. Metelmann, M. H. Matheny, F. Marquardt, A. A. Clerk, and O. Painter, Generalized nonreciprocity in an optomechanical circuit via synthetic magnetism and reservoir engineering, Nat. Phys. 13, 465 (2017).

[70] J. Gallego, S. Ghosh, S. K. Alavi, W. Alt, M. MartinezDorantes, D. Meschede, and L. Ratschbacher, Highfinesse fiber fabry-perot cavities: Stabilization and mode matching analysis, Appl. Phys. B 122, 47 (2016).

[71] J. Gallego, W. Alt, T. Macha, M. Martinez-Dorantes, D. Pandey, and D. Meschede, Strong Purcell Effect on a Neutral Atom Trapped in an Open Fiber Cavity, Phys. Rev. Lett. 121, 173603 (2018).

[72] T. G. Tiecke, K. P. Nayak, J. D. Thompson, T. Peyronel, N. P. deLeon, V. Vuletić, and M. D. Lukin, Efficient fiberoptical interface for nanophotonic devices, Optica 2, 70 (2015).

[73] R. Yamamoto, J. Kobayashi, T. Kuno, K. Kato, and Y. Takahashi, An ytterbium quantum gas microscope with narrow-line laser cooling, New J. Phys. 18, 023016 (2016).

[74] S. Saskin, J. T. Wilson, B. Grinkemeyer, and J. D. Thompson, Narrow-line cooling and imaging of ytterbium atoms in optical tweezer array, arXiv:1810.10517v1 (2018).

[75] A. Cooper, J. P. Covey, I. S. Madjarov, S. G. Porsev, M. S. Safronova, and M. Endres, Alkaline-earth Atoms in Optical Tweezers, Phys. Rev. X 8, 041055 (2018).

[76] M. A. Norcia, A. W. Young, and A. M. Kaufman, Microscopic Control and Detection of Ultracold Strontium in Optical-tweezer Arrays, Phys. Rev. X 8, 041054 (2018).

[77] Y. Meng, A. Dareau, P. Schneeweiss, and A. Rauschenbeutel, Near-ground-state Cooling of Atoms Optically Trapped $300 \mathrm{~nm}$ Away from a Hot Surface, Phys. Rev. X 8, 031054 (2018).

[78] K. Beloy, J. A. Sherman, N. D. Lemke, N. Hinkley, C. W. Oates, and A. D. Ludlow, Determination of the $5 d 6 s^{3} D_{1}$ state lifetime and blackbody-radiation clock shift in $\mathrm{Yb}$, Phys. Rev. A 86, 051404 (2012).

[79] Z.-M. Tang, Y.-M. Yu, J.-G. Li, and C.-Z. Dong, Magic wavelengths for the ${ }^{1} S_{0}$, and ${ }^{3} P_{1}$ transition in ytterbium atom, J. Phys.: Conf. Ser. 875, 022050 (2017).

[80] F. Scazza, PhD thesis, Ludwig-Maximilians-Universitat Munchen, 2015.

[81] H. Bernien, S. Schwartz, A. Keesling, H. Levine, A. Omran, H. Pickler, S. Choi, A. S. Zibrov, M. Endres, M. Greiner, V. Vuletíc, and M. D. Lukin, Probing many-body dynamics on a 51-atom quantum simulator, Nature 551, 579 (2017).

[82] R. Ritter, N. Gruhler, W. Pernice, H. Kübler, T. Pfau, and R. Löw, Atomic vapor spectroscopy in integrated photonic structures, Appl. Phys. Lett. 107, 041101 (2015).

[83] R. Ritter, N. Gruhler, W. H. P. Pernice, H. Kübler, T. Pfau, and R. Löw, Coupling thermal atomic vapor to an integrated ring resonator, New J. Phys. 18, 103031 (2016).

[84] A. Derevianko, S. G. Porsev, and J. F. Babb, Electric dipolepolarizabilities at imaginary frequencies for hydrogen, the alkali-metal, alkaline-earth, and noble gas atoms, Atomic Data Nucl. Data Tables 96, 323 (2010).

[85] P. E. Barclay, K. Srinivasan, and O. Painter, Nonlinear response of silicon photonic crystal microresonators excited via an integrated waveguideand fiber taper, Opt. Express 13, 801 (2005).

[86] T. J. Johnson, M. Borselli, and O. Painter, Self-induced optical modulation of the transmission through a highq silicon microdisk resonator, Opt. Express 14, 817 (2006).

[87] K. Bücher, J. Bruns, and H. G. Wagemann, Absorption coefficient of silicon: An assessment of measurements and the simulation of temperature variation, J. Appl. Phys. 75, 1127 (1994).

[88] J.-H. Kim, C. J. K. Richardson, R. P. Leavitt, and E. Waks, Two-photon interference from the far-field emission of chip-integrated cavity-coupled emitters, Nano Lett. 16, 7061 (2016).

[89] P. Komar, E. M. Kessler, M. Bishof, L. Jiang, A. S. Sorensen, J. Ye, and M. D. Lukin, A quantum network of clocks, Nat. Phys. 10, 582 (2014).

[90] D. A. Steck, Quantum and atom optics, http://steck.us/ teaching.

[91] D. S. Barker, N. C. Pisenti, B. J. Reschovsky, and G. K. Campbell, Three-photon process for producing a degenerate gas of metastable alkaline-earth-metal atoms, Phys. Rev. A 93, 053417 (2016).

[92] M. M. Boyd, T. Zelevinsky, A. D. Ludlow, S. Blatt, T. Zanon-Willette, S. M. Foreman, and J. Ye, Nuclear spin effects in optical lattice clocks, Phys. Rev. A 76, 022510 (2007).

[93] A. V. Gorshkov, A. M. Rey, A. J. Daley, M. M. Boyd, J. Ye, P. Zoller, and M. D. Lukin, Alkaline-earth-metal Atoms as Few-qubit Quantum Registers, Phys. Rev. Lett. 102, 110503 (2009).

[94] L. Robledo, L. Childress, H. Bernien, B. Hensen, P. F. A. Alkemade, and R. Hanson, High-fidelity projective readout of a solid-state spin quantum register, Nature 477, 574 (2011).

[95] J. Calsamiglia and N. Lütkenhaus, Maximum efficiency of a linear-optical bell-state analyzer, Appl. Phys. B 72, 67 (2001).

[96] A. Napoli and J. Pedro, Getting the most from currently deployed optical fiber infrastructure, Fibre Systems, Viewpoint (2017).

[97] N. Sangouard, R. Dubessy, and C. Simon, Quantum repeaters based on single trapped ions, Phys. Rev. A 79, 042340 (2009).

[98] F. Marsili, V. B. Verma, J. A. Stern, S. Harrington, A. E. Lita, T. Gerrits, I. Vayshenker, B. Baek, M. D. Shaw, R. P. Mirin, and S. W. Nam, Detecting single infrared photons with 93\% system efficiency, Nat. Photonics 7, 210 (2013).

[99] T. Legero, T. Wilk, A. Kuhn, and G. Rempe, Timeresolved two-photon quantum interference, Appl. Phys. B 77, 797 (2003). 
[100] X.-J. Wang, B. Jing, P.-F. Sun, C.-W. Yang, Y. Yu, V. Tamma, X.-H. Bao, and J.-W. Pan, Experimental Timeresolved Interference with Multiple Photons of Different Colors, Phys. Rev. Lett. 121, 080501 (2018).

[101] T. Steinmetz, Y. Colombe, D. Hunger, T. W. Hänsch, A. Balocchi, R. J. Warburton, and J. Reichel, Stable fiberbased Fabry-Pérot cavity, Appl. Phys. Lett. 89, 111110 (2006).

[102] Y. Colombe, T. Steinmetz, G. Dubois, F. Linke, D. Hunger, and J. Reichel, Strong atom-field coupling for Bose-Einstein condensates in an optical cavity on a chip, Nature 450, 272 (2007).

[103] D. Hunger, T. Steinmetz, Y. Colombe, C. Deutsch, T. W. Hänsch, and J. Reichel, A fiber Fabry-Perot cavity with high finesse, New J. Phys. 12, 065038 (2010).

[104] U. I. Safronova and M. S. Safronova, Third-order relativistic many-body calculations of energies, transition rates, hyperfine constants, and blackbody radiation shift in ${ }^{171} \mathrm{Yb}^{+}$, Phys. Rev. A 79, 022512 (2009).

[105] V. A. Dzuba and V. V. Flambaum, Calculation of nuclearspin-dependent parity nonconservation in $\mathrm{s}-\mathrm{d}$ transitions of $\mathrm{Ba}^{+}, \mathrm{Yb}^{+}$, and $\mathrm{Ra}^{+}$ions, Phys. Rev. A 83, 052513 (2011).

[106] B. K. Sahoo and B. P. Das, Parity nonconservation in ytterbium ion, Phys. Rev. A 84, 010502 (2011).

[107] S. G. Porsev, M. S. Safronova, and M. G. Kozlov, Correlation effects in $\mathrm{Yb}^{+}$and implications for parity violation, Phys. Rev. A 86, 022504 (2012).

[108] T. Feldker, H. Fürst, N. V. Ewald, J. Joger, and R. Gerritsma, Spectroscopy of the ${ }^{2} S_{1 / 2} \rightarrow{ }^{2} P_{3 / 2}$ transition in $\mathrm{Yb}$ II: Isotope shifts, hyperfine splitting, and branching ratios, Phys. Rev. A 97, 032511 (2018).

[109] T. Schneider, E. Peik, and C. Tamm, Sub-hertz Optical Frequency Comparisons between Two Trapped ${ }^{171} \mathrm{Yb}^{+}$ Ions, Phys. Rev. Lett. 94, 230801 (2005).

[110] M. Steiner, H. M. Meyer, C. Deutsch, J. Reichel, and M. Köhl, Single Ion Coupled to an Optical Fiber Cavity, Phys. Rev. Lett. 110, 043003 (2013).

[111] M. Zwerger, B. P. Lanyon, T. E. Northup, C. A. Muschik, W. Dür, and N. Sangouard, Quantum repeaters based on trapped ions with decoherence-free subspace encoding, Quantum Sci. Technol. 2, 044001 (2017). 